\title{
Anatomy of the Distal Incus in Humans
}

\author{
Wade Chien, ${ }^{1,2}$ Clarinda Northrop, ${ }^{1,3}$ Stephen Levine, ${ }^{3}$ Ben Z. Pilch, ${ }^{1,5}$ William T. Peake, ${ }^{1,4}$ \\ John J. Rosowski, ${ }^{1,2,4}$ and Saumil N. Merchant ${ }^{1,2}$ \\ ${ }^{1}$ Department of Otolaryngology, Massachusetts Eye $\mathcal{E}$ Ear Infirmary, 243 Charles Street, Boston, MA 02114, USA \\ ${ }^{2}$ Department of Otology and Laryngology, Harvard Medical School, Boston, MA, USA \\ ${ }^{3}$ The Temporal Bone Foundation, Boston, MA, USA \\ ${ }^{4}$ Research Laboratory of Electronics, Massachusetts Institute of Technology, Cambridge, MA, USA \\ ${ }^{5}$ Department of Pathology, Massachusetts General Hospital, Boston, MA, USA
}

Received: 14 January 2009; Accepted: 18 June 2009; Online publication: 15 August 2009

\begin{abstract}
The anatomy of the distal incus, including the lenticular process, was examined in histological sections from 270 normal cadaveric human temporal bones aged between less than 1 month and 100 years. All but nine of these sectioned specimens showed signs of a bony connection between the long process of the incus and the flattened plate of the lenticular process, and in 108 specimens a complete bony attachment was observed in a single $20 \mu \mathrm{m}$ section. In these 108 ears, the bony lenticular process consisted of a proximal narrow "pedicle" connected to a distal flattened "plate" that forms the incudal component of the incudo-stapedial joint. A fibrous joint capsule extended from the stapes head to the pedicle of the lenticular process on all sides, where it was considerably thickened. Three-dimensional reconstructions made from serial $20 \mu \mathrm{m}$ sections of four bones provided views from all directions that easily convey the anatomical features of this region. Morphometric measurements of the bony architecture of the distal incus in 103 temporal bones were made, including lengths and cross-sectional areas, estimates of the percentage of lacunae containing osteocytes, and the degree of bone resorption. These measurements, analyzed as a function of age, provided an anatomic description over a large age range that can serve as a normal baseline against which structural
\end{abstract}

Correspondence to: Wade Chien · Department of Otolaryngology · Massachusetts Eye \& Ear Infirmary - 243 Charles Street, Boston, MA 02114, USA. Telephone: +1-617-9212964; email: wadechien@ hotmail.com pathology can be compared. Although none of the bony dimensions showed significant age dependence, the estimated percentage of bony lacunae that contain osteocytes decreased significantly with age. The results have implications for the roles of specific components on the coupling of ossicular motion across the incudo-stapedial joint, and provide insights regarding bone resorption at the level of the distal incus, which occurs clinically in some patients with chronic otitis media or after stapedectomy.

Keywords: incus, lenticular process, incus necrosis, middle-ear mechanics, incudo-stapedial joint

\section{INTRODUCTION}

The incus is a member of the ossicular chain in mammalian middle ears that couples sound signals from the tympanic membrane to the inner ear. The long process of the incus is connected to the head of the stapes by the lenticular process. This region, which we refer to as the "distal incus", is frequently affected by middle-ear disease. For example, the distal incus is often a site of bone erosion in chronic otitis media. Also, in patients who have undergone stapedectomy, resorption of the distal incus is a common cause of recurrent conductive hearing loss. Despite its clinical significance, the anatomy and histology of the human distal incus, including the lenticular process, have not been described systematically. In many drawings of the incus, as exemplified in Figure 1, the 


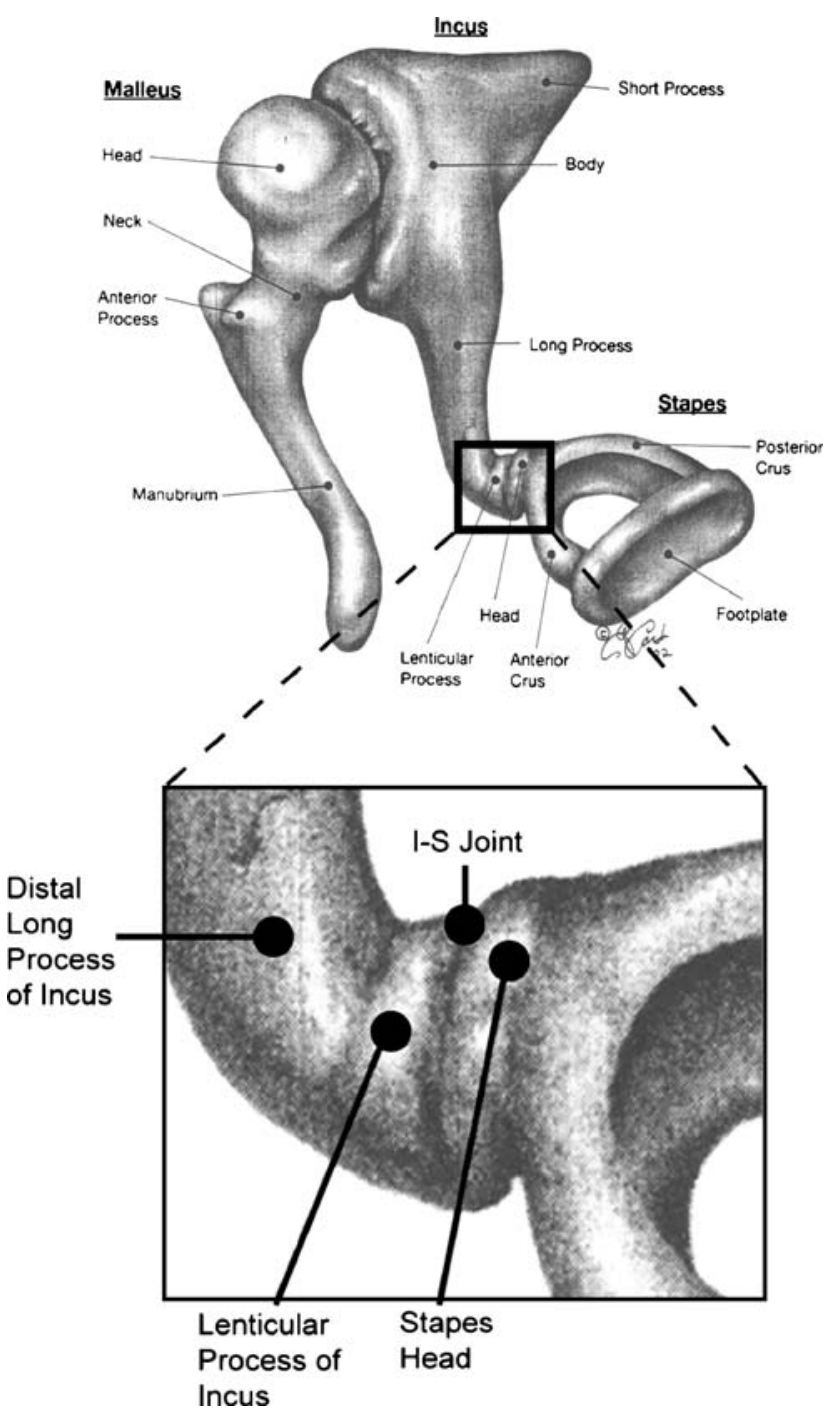

FIG. 1. Drawing of the human ossicular chain taken from the Glasscock-Shambaugh Surgery of the Ear textbook (5th edition, 2005, B.C. Decker; reproduced with permission). Note that the lenticular process is depicted as a cylindrical disc, and the diameter of the incus long process is similar in size to the diameter of the lenticular process.

lenticular process is depicted as a cylindrical disc with a similar diameter as the adjacent long process (Glasscock and Gulya 2005). In contrast, in a recent anatomic study of the incudo-stapedial (I-S) joint in cats, Funnell et al. (2005) concluded that the bony lenticular process consists of a flattened "plate" attached to the distal long process by a narrow "pedicle". Funnell and colleagues (2005, 2006) also presented a high-resolution radiological image that implies a similar configuration in the human ear.

The goal of the present study is to systematically describe the normal anatomy of the human distal incus using histological sections in the archival temporal bone collection at the Massachusetts Eye \& Ear Infirmary. In addition to qualitative descriptions of the anatomy, three-dimensional (3-D) computer reconstructions and morphometric measurements were performed to display the complexities of the anatomy in this region. The effects of age and gender on the bony architecture of the distal incus were also evaluated. The results have implications for understanding the basic mechanisms of sound transmission through the middle ear, and provide a normal baseline against which structural pathology can be evaluated.

\section{MATERIAL AND METHODS}

The temporal bone collection at the Massachusetts Eye \& Ear Infirmary contains specimens prepared in a standard manner for light microscopy (Schuknecht 1993). The temporal bones are serially sectioned in either the axial or vertical plane at a thickness of $20 \mu \mathrm{m}$, and every tenth section is stained with hematoxylin and eosin (H\&E).

The temporal bones selected for this study were from individuals with no history of middle-ear disease and with normal anatomy of the middle ear. Specimens with the following diagnosis were excluded: congenital syndromes affecting the ear, temporal bone fractures, inflammatory inner-ear disorders, temporal bone tumors, history of otologic surgery, or radiation exposure to the temporal bone.

Histological sections from 353 normal temporal bones were examined. Eighty-three bones were excluded due to artifacts of removal or preparation involving the incudo-stapedial joint. Of the 270 remaining bones, only nine did not show evidence of a bony connection between the incus long process and the lenticular plate, i.e., there were no signs of a pedicle between the incus long process and the plate of the lenticular process. (Note-only the stained sections [i.e., every tenth section] were examined in this study. The intervening unstained sections were not available to determine definitively the presence or absence of such a bony connection.)

Of the 261 remaining bones with evidence of a bony connection between the incus long process and the lenticular plate, 108 bones were selected for further detailed analysis based on the presence of a continuous bony pedicle between the incus long process and the lenticular plate in a single $20 \mu \mathrm{m}$ section (Figs. 2, 3, 4, and 5). One hundred and three of these bones had been sectioned in the axial (horizontal) plane, and five bones in the vertical plane. The age of the donors whose specimens were examined ranged from 1 day to 100 years, with ten bones between 0 and 1 year, six bones between 1 and 2 years, five bones between 2 and 10 years, and seven 
A

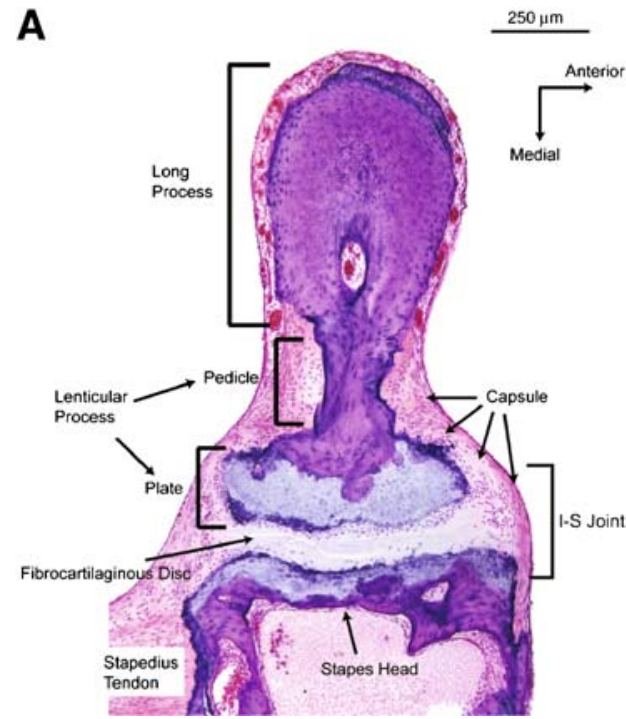

FIG. 2. A A representative histological section of the distal incus in the axial (horizontal) plane (from a 22-year-old male, H\&E stain), showing the distal long process with its connection to the pedicle and the plate of the lenticular process. Note the narrow bony pedicle

to 11 bones for each decade between 10 and 100 years. The average age was 47 years.

Three methods were used to examine the anatomy of the distal incus in these 108 temporal bones:

1. Histological examination: the stained sections (every tenth section) were examined in each temporal bone to obtain a general description of the anatomy.

2. 3-D reconstruction: in four temporal bones (aged 2 months, 14 years, 52 years, and 81 years), each and every section $(20 \mu \mathrm{m}$ thick) through the distal
B

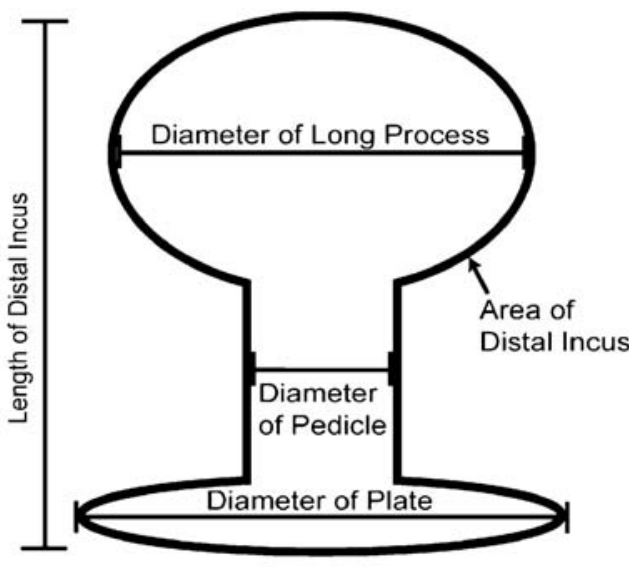

of the lenticular process and the abundant connective tissue surrounding it. B Structural parameters of the distal incus measured in our specimens. See Table 1 for measurement results.

incus was stained to allow for 3-D reconstruction. Each section was digitized and imported into 3-D imaging programs, Maya software (Alias Systems Corp., Toronto, Canada), and Amira software (Mercury Computer Systems/TGS, San Diego, CA, USA). A virtual 3-D surface model of the bony distal incus was reconstructed for each of the four bones.

3. Morphometric measurements: the following structural parameters were measured in each of the 103 bones sectioned in the axial plane (Fig. 2A, B, and Table 1): the length and area of the distal incus

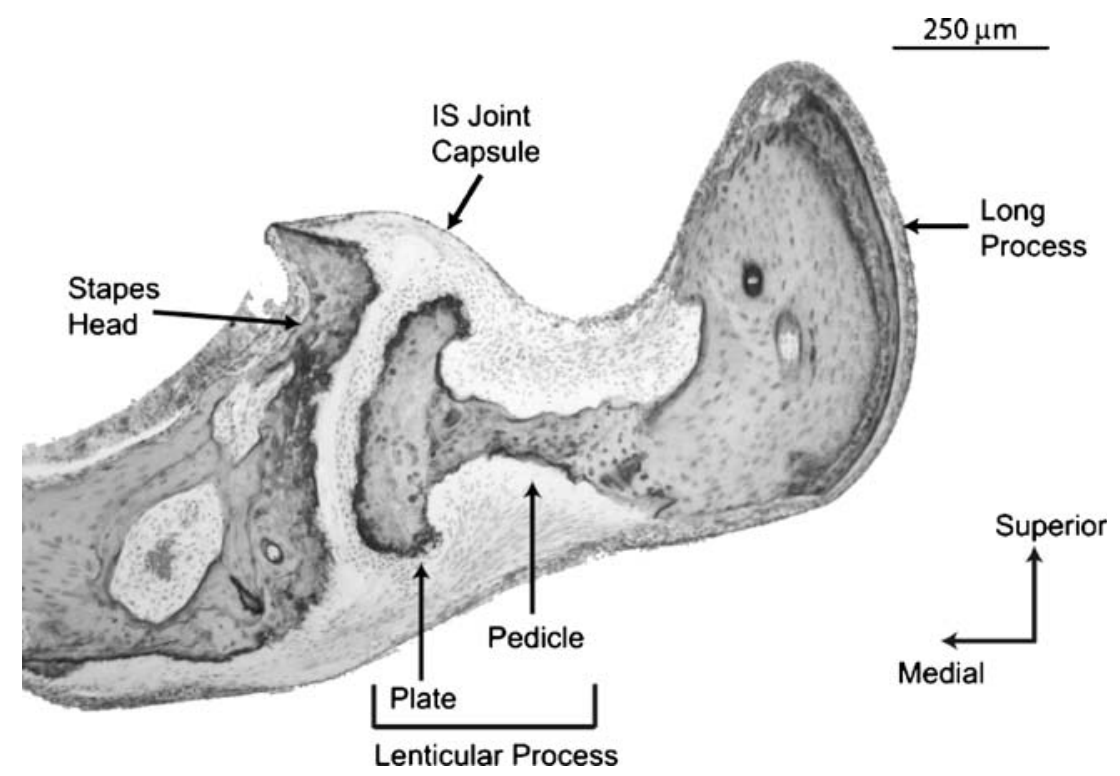

FIG. 3. A histological section in the vertical plane (from a 33-year-old female) showing the narrow bony pedicle and the widened plate. 


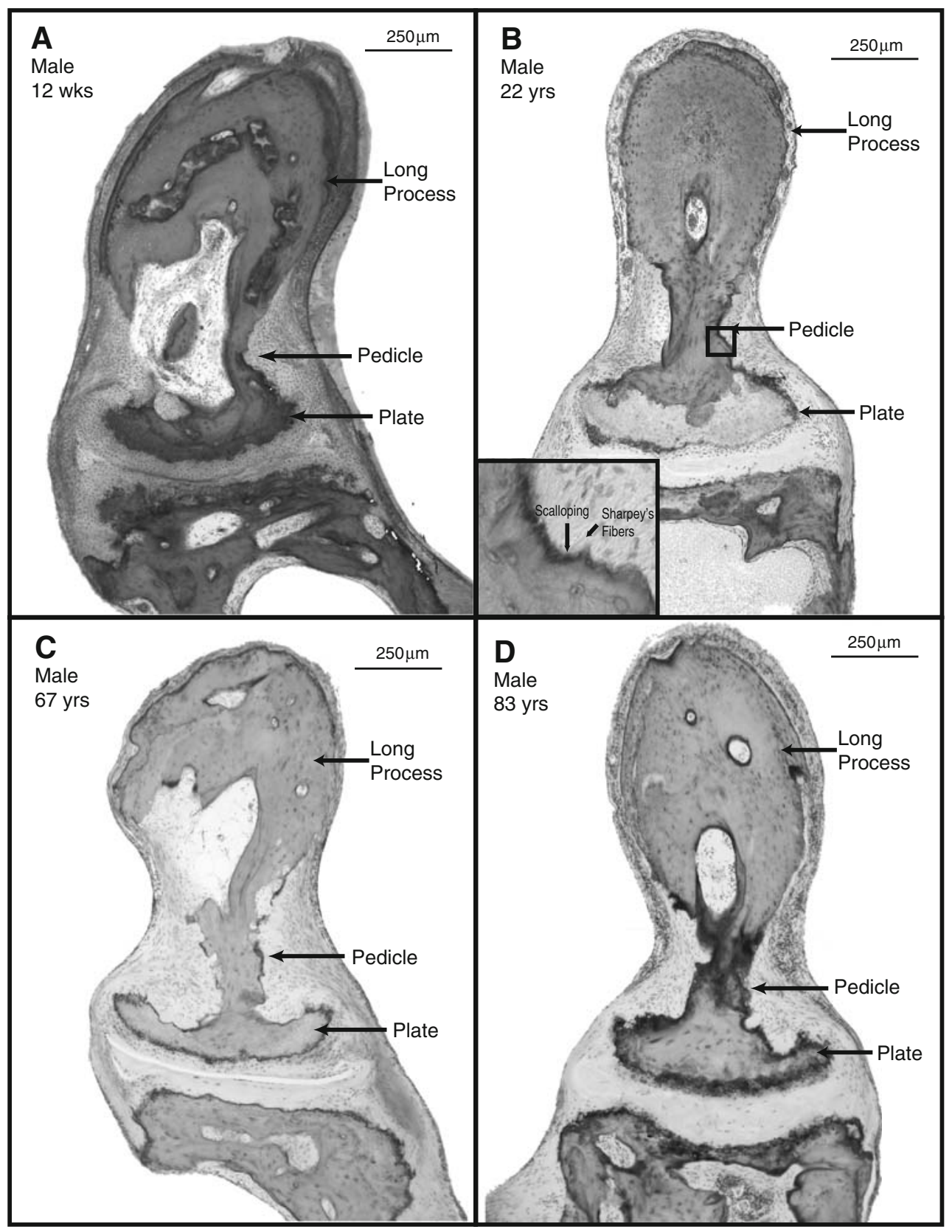

FIG. 4. Representative histological sections in the axial plane of the distal incus from four individuals at various ages. In each section, a narrow bony pedicle is seen connecting the long process to the plate of the lenticular process. The inset in (B) shows the area outlined in the box under higher magnification $(\times 100)$. Note the slight scalloping of the bony pedicle as the capsular fibers insert. These fibers travel for a short distance into the bone, and are termed Sharpey's fibers. including the lenticular process, the diameter of the distal long process, the diameters of the pedicle and plate of the lenticular process, and the total area of tissue within the bony outer border of the distal incus that was not bone, such as vascular channels, areolar soft tissue, and areas of bone resorption (designated as "area of non-bone"). In addition, based on inspection of the bone throughout the section, a visual estimate was made of the percentage of osteocyte-containing lacunae (the estimates were stratified into four bins $=75-100 \%$, $50-75 \%, 25-50 \%$, and $0-25 \%)$. The histological sections used for the morphometric measurements were digitized using Metamorph software (Molecular Devices Corporation, Sunnyvale, CA, USA), and the morphometric measurements were performed using Image J Software (National Institutes of Health, Bethesda, MD, USA). Statistical analysis was performed using Excel software (Microsoft Corporation, Redmond, WA, USA). 


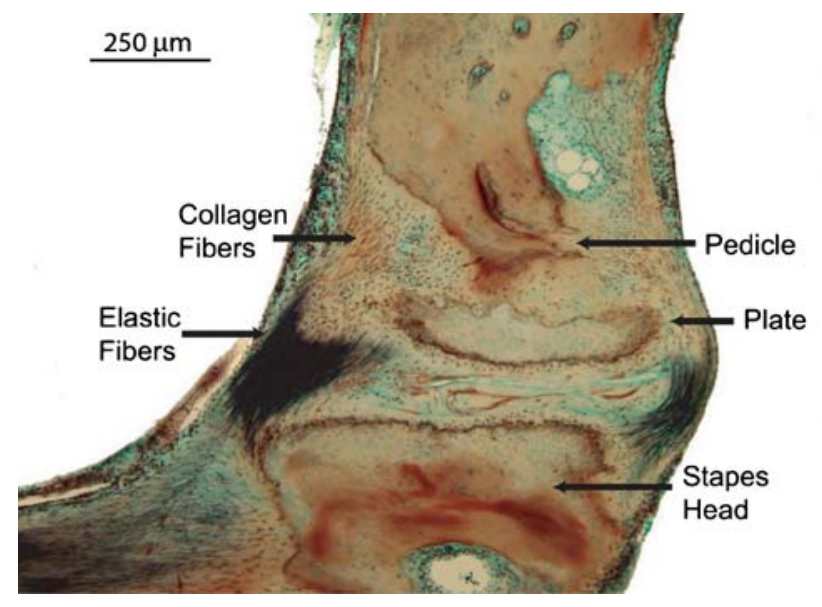

FIG. 5. Composition of fibers of I-S joint capsule using Movat's Pentachrome Stain (from a 22-year-old male). Elastic fibers are stained black, and collagen fibers are stained yellow/orange. Note the prominent elastic tissue component of the I-S joint capsule around the joint space. This slide is representative of all three specimens.

In three specimens, selected sections were stained using Russell's modification of Movat's pentachrome stain to examine the fibers within the capsule of the I$\mathrm{S}$ joint. This stain allowed us to differentiate between elastic fibers (rich in elastin) which stain black, and collagen fibers which stain yellow/orange (Luna 1972).

\section{RESULTS}

\section{Qualitative histological descriptions (Figs. 2, 3, 4, and 5)}

The lenticular process has a bony pedicle that is quite narrow, and a flattened osteocartilaginous plate that forms the incudal portion of the I-S joint. This anatomic configuration is seen both in axial (Fig. 2A) and vertical (Fig. 3) sections. The narrow pedicle and flattened plate configuration was present in 107/108 bones at all ages (Fig. 4); in one bone (aged 5 years), the diameters of the pedicle and plate were about equal. The presence of the narrow pedicle and flattened plate configuration of the lenticular process across all age groups indicates that this configuration is the normal anatomy.

The I-S joint is a diarthrodial joint. It consists of a narrow slit-like space lined by synovium lying between the hyaline cartilaginous surfaces of the lenticular plate and the stapes head, and a fibrous capsule on the outer surface of the joint. A fibrocartilaginous disc was visible in between the synovial surfaces of the I-S joint (Fig. 2A) in about $80 \%$ of our specimens.

The capsule of the I-S joint is specialized (Fig. 2A): the capsule attaches to a narrow area along the outer edge of the stapes head, thickens considerably at the level of the pedicle, and is attached to the pedicle and plate over a broad surface area. The bony surface of the lenticular process often appears slightly scalloped where the capsular fibers attach; such scalloping has been cited as evidence for bone resorption (Baron 1963; Bellucci and Wolff 1966). However, when viewed under high magnification, it is evident that the scalloping occurs where capsular fibers attach onto the bony pedicle (Fig. 4B). These fibers penetrate directly into bone, and constitute Sharpey's fibers (Ross and Pawlina 2005), similar to those seen in other joints where capsular fibers are attached to a bony surface. None of these scalloped areas contained osteoclasts, which play an important role in bone metabolism and are responsible for bone resorption. The lack of osteoclasts supports the notion that the slight degree of scalloping is not due to bone resorption but is a feature of the normal anatomy.

The I-S joint capsule is highly cellular, containing cells with either spindle- or oval-shaped nuclei, as well as connective tissue fibers. Russell's modification of Movat's Pentachrome Stain showed that the fibers immediately surrounding the I-S joint space are composed mainly of elastic fibers, whereas the fibers attaching to the pedicle of the lenticular process are mainly collagen fibers (Fig. 5). The presence of elastic

\section{TABLE 1}

\begin{tabular}{|c|c|c|c|c|c|c|}
\hline \multicolumn{7}{|c|}{ Morphometric measurements made from histological sections } \\
\hline Measured parameters & $N$ & Mean & Range & Std. dev & $r$ & Statistical significance \\
\hline Length of distal incus $(\mu \mathrm{m})$ & 103 & 1,200 & $653-1,717$ & 203 & 0.18 & No \\
\hline Area of distal incus $\left(\mu \mathrm{m}^{2}\right)$ & 103 & 560,000 & $230,321-918,710$ & 167,204 & 0.13 & No \\
\hline Diameter of long process $(\mu \mathrm{m})$ & 103 & 630 & $260-1,100$ & 134.43 & 0.14 & No \\
\hline Diameter of pedicle $(\mu \mathrm{m})$ & 103 & 260 & $65-530$ & 104 & 0.01 & No \\
\hline Diameter of plate $(\mu \mathrm{m})$ & 103 & 710 & 475-1,099 & 108 & -0.02 & No \\
\hline Ratio of non-bone/total area & 103 & 0.12 & $0.01-0.52$ & 0.1 & 0.21 & Yes \\
\hline Percentage of osteocyte-filled lacunae (\%) & 103 & 43.4 & $0-100$ & NA & -0.89 & Yes \\
\hline
\end{tabular}

$N$ number of temporal bones examined. Mean the mean for each measured parameter. Range the maximum and minimum for each measured parameter. Std. dev standard deviation for each measured parameter. $r$ correlation coefficient. Statistical significance is defined as $p<.05$ 
fibers in the I-S joint is consistent with previous studies by Davies (1948) and Harty (1953). The capsule is thickest at the pedicle and relatively narrow at the level of the plate. This inverse relationship between capsular and bony thickness produces a relatively uniform thickness of the combination of bone and capsule.

The stapedius tendon inserted entirely onto the head of stapes in most of the specimens; but in seven out of 103 axial specimens (7\%), it also partially inserted into the I-S joint capsule. The number of vascular channels seen in a cross section of the incus long process varies from one to several; these Haversian canals contain the blood vessels that supply the incus. The blood vessels within the long process communicate freely with a rich mucosal vascular plexus. A vascular channel is usually seen within the pedicle of the lenticular process and can sometimes be seen within the plate.

\section{Gross vs. histologic anatomy}

It is instructive to compare a representative histological section of the distal incus to a view of the same area as seen with an operating microscope (Fig. 6A-C). In the histological section (Fig. 6A), both the bony distal long process of the incus and the plate of the lenticular process clearly have larger diameters than the narrower bony pedicle of the lenticular process. However, when examined under an operating microscope (Fig. 6B and $\mathrm{C})$, the diameters of the distal long process and the pedicle region appear about the same. This difference in appearance is a result of the thickening of the soft tissue of the I-S joint capsule around the narrow pedicle (noted above), which obscures the narrowing of the pedicle in the surgical view.

\section{Three-dimensional reconstruction of the bony surface of the distal incus}

Three-dimensional surface reconstructions of the bony distal incus were made from serial sections of four temporal bone preparations (Fig. 7). The four preparations were from individuals aged 2 months, 14 years, 52 years, and 81 years. The images have been rotated so that the components of the distal incus are oriented similarly in the four images. With these computational reconstructions, the bony anatomy can be observed from all directions. In each case, the three components of the distal incus are clearly distinguishable, with the distal long process connected to the plate by the bony pedicle. The pedicle is narrower than both the long process and the plate. Both Sharpnell (1832) and Funnell et al. (2005) suggest that the anterior-posterior dimension of the pedicle is much wider than its superior-inferior dimension. Since most of our observations were based

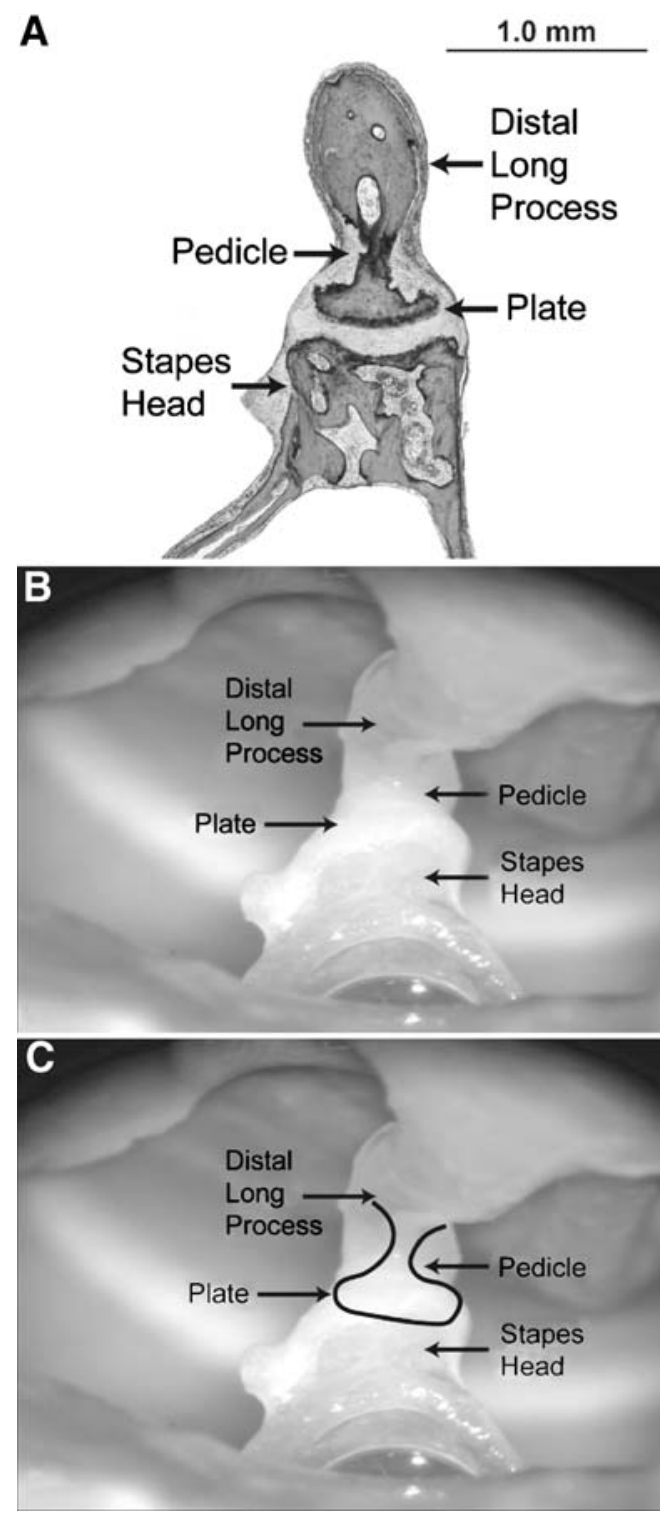

FIG. 6. Comparison of histological and gross images of the distal incus. In the histological image (A), the narrow bony pedicle is readily appreciated. However, in the gross images (B and C), taken from another specimen, the diameter of the pedicle looks similar to the diameters of the distal long process and the plate. The outlines drawn in $(\mathbf{C})$ represent an estimate of the shape of the bony distal incus based on our observations of other specimens.

on single anatomical sections through the pedicle, we have little data on this issue. However, the four reconstructions from serial sections suggest there is a significant variability in this feature. In Fig. 7B and D, the pedicles are much wider in the anterior-posterior dimension than they are in the superior-inferior dimension, while the pedicles in Fig. 7A and C appear more circular in cross section. The plate is roughly oval in its parasagittal plane; at its junction with the pedicle, the plate's lateral concavity is reminiscent of the cap of a mushroom. The distal long process 
A Male, 2 months

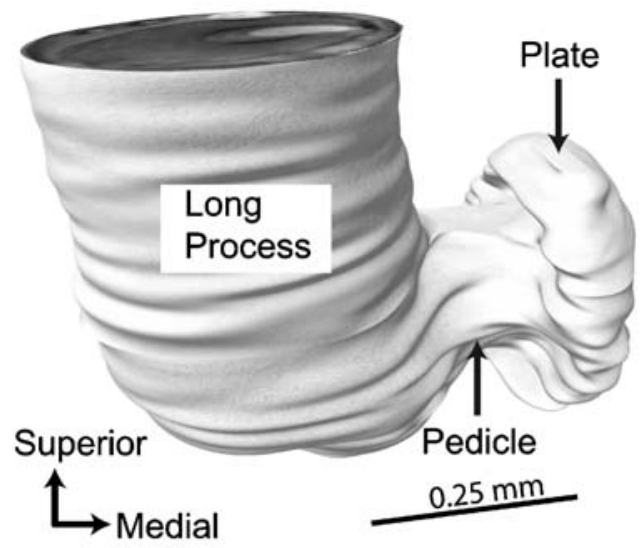

C Female, 52 years

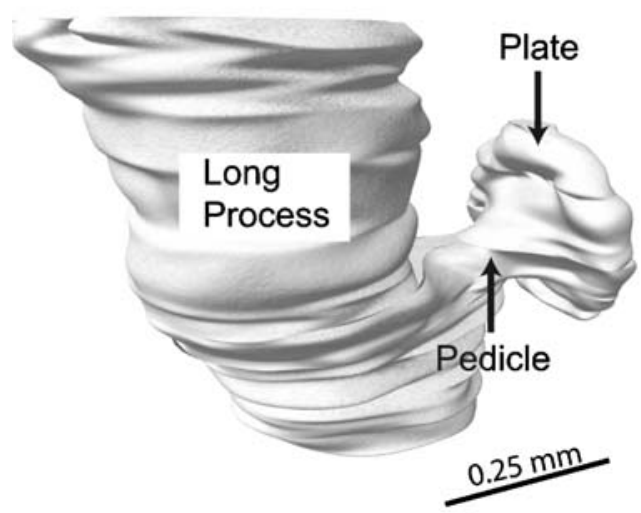

FIG. 7. 3-D reconstructions of the distal incus in four subjects. In all cases, the lenticular process consists of a narrow bony pedicle connecting to an expanded plate. Please note that the four panels are

extends inferiorly beyond its junction with the pedicle tapering to a rounded inferior surface. The 3-D model from the 14-year-old specimen is available as a free download from our website (http://temporalbone consortium.org/educational-resources/3d-models). The 3-D data sets for the other three specimens are also available from author $\mathrm{CN}$ upon request.

4. Morphometric measurements and dependence on age and gender of the anatomy of the distal incus

The results of morphometric measurements of the distal incus (Fig. 2B) are shown in Table 1. The mean diameter of the bony pedicle $(0.26 \mathrm{~mm})$ is less than half of the mean diameter of the distal long process $(0.63 \mathrm{~mm})$ and also less than half of the mean diameter of the plate $(0.71 \mathrm{~mm})$. None of the measured dimensions show significant changes with age except for the ratio of the area of non-bone to the total area of the incus and the percentage of lacunae containing osteocytes. There was a clear trend towards

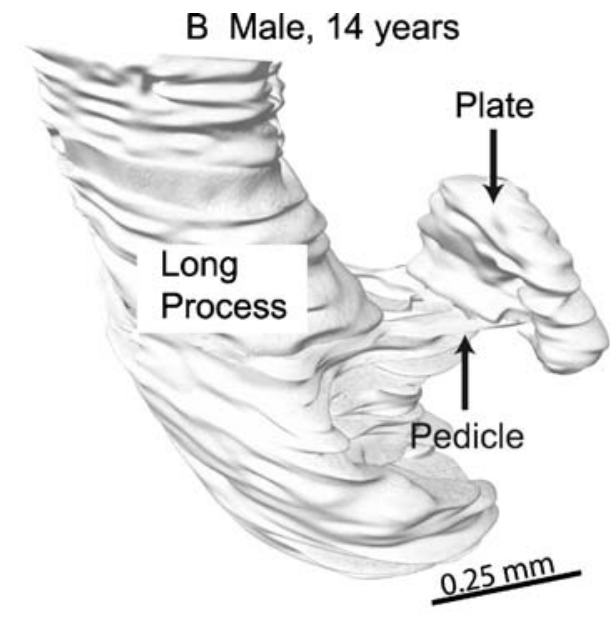

D Male, 81 years

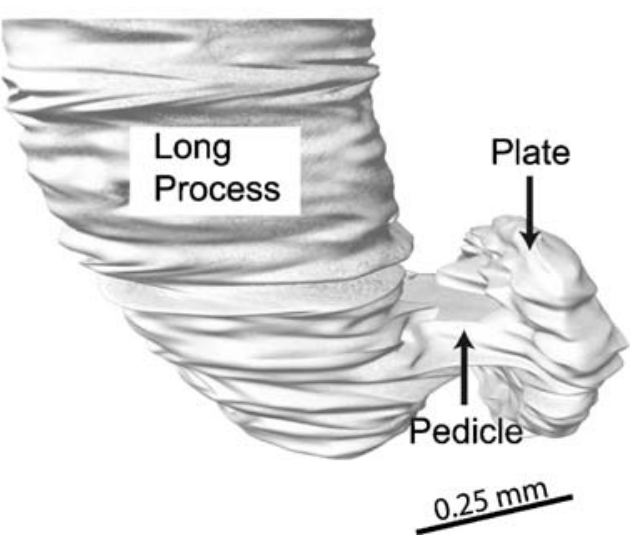

not on precisely the same scale. The scale bars represent the projection of a $0.25-\mathrm{mm}$ length aligned in the medial-lateral direction in each panel.

dropout of osteocytes as a function of advancing age (Fig. 8). However, a corresponding increase in resorption of bone (as measured by the ratio of nonbone area to the total area of distal incus), though

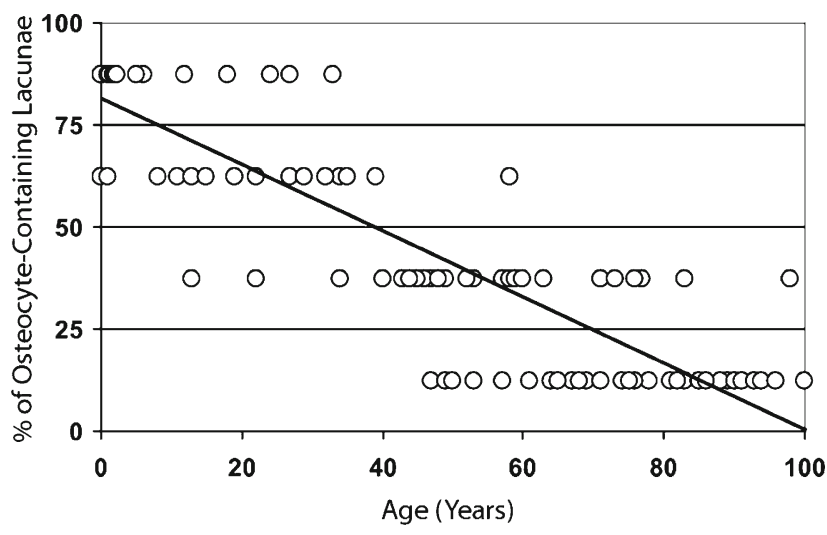

FIG. 8. The relationship between the percentage of osteocytecontaining lacunae and age. The straight line is the regression line based on the data points. Also see Table 1. 
statistically significant $(p<0.05)$, was small (on average from 0.1 at infancy to 0.15 at $90-100$ years of age), and probably not clinically significant.

The effect of gender on distal incus morphology was also examined (Table 2). Of the 103 axial specimens examined, 63 specimens were male, and 40 specimens were female. We found significant differences in the area of distal incus $\left(590,000 \mu \mathrm{m}^{2}\right.$ for male, and $520,000 \mu \mathrm{m}^{2}$ for female, $\left.p=0.04\right)$ and in the diameter of the plate $(730 \mu \mathrm{m}$ for male, and $670 \mu \mathrm{m}$ for female, $p=0.004)$. These differences are consistent with an overall size difference between male and female.

\section{DISCUSSION}

\section{Lenticular process as the "fourth" ossicle?}

Over the last hundred years, occasional reports have supported the idea that the number of ossicles in the mammalian middle ear is four, in contradiction to the prevalent view that the ossicular chain consists of three bones: malleus, incus, and stapes (see Funnell et al. 2005 for a concise review). The four-ossicle interpretation is based on the idea that the small lenticular plate, which abuts the head of the stapes, is not connected by bone to the long process of the incus. Names proposed for this "fourth ossicle" have included "osselet de Sylvius" and os lenticulare (Valsalva 1704); those who have concluded that this structure is connected to the long process of the incus by a bony neck have called the bony, two-part structure the Sylvian apophysis, or epiphysis of the incus, or lenticular process (Sharpnell 1832). The "three versus four" ossicle contention has an impact beyond the ear; the identity of "the smallest bone in the human body" is dependent on whether the lenticular plate qualifies to enter this competition among independent bones.

(a) Evidence for three ossicles:

Sharpnell (1832) used both experimental and observational approaches to study the ossicles:
"I subjected four temporal bones to a full process of maceration, that all ligamentous connexions might be destroyed, and the bones separated without violence. But in each of these instances, and in numerous temporal bones of various animals, no separation of the lenticulare from the incus could be effected by maceration. The same circumstance may ... occasionally be obtained from temporal bones that have been long buried, and in which a more complete process of decomposition has taken place than can usually be obtained by maceration. The appearance of the connexion of the reputed os lenticulare to the incus, is that of a distinct neck or process of bone, (that) spreads out into an oval plate, which forms the articulation with the head of the stapes."

Sharpnell concluded: "the os lenticulare is to be struck out of the list of the bones and should be considered merely a process - namely the process lenticularis of the os incus. The os stapes, and not the os lenticulare is the smallest bone in the body. The number of ossicula auditus is three, not four." (Sharpnell 1832). Views of others who supported Sharpnell's conclusion are summarized by Funnell et al. (2005).

(b) Evidence for four ossicles:

Histological sections from human cadaver ears might be expected to provide direct evidence to settle the issue through demonstration of a bony connection (or lack of a bony connection) of the os lenticulare to the distal long process of the incus. However, interpretations of histological results have not been uniformly supportive of one conclusion. A photograph of a histologic section in Wolff et al. (1971, pp. 96-97) appears to support union of the lenticular process to the incus, showing a "narrownecked attachment of the lenticular process to the descending crus of the incus". However, this caption also states that "it is known that the lenticular process is sometimes a separate bone" (with no evidence cited), thereby supporting the idea that three or four ossicles can occur. It does, however, seem possible, that sections with no bony connection may fail to contain the "narrow-necked process" even though it is present in near-by sections.

\section{TABLE 2}

Comparison of morphometric measurements between male and female

\begin{tabular}{lllll}
\hline Measured parameter & Male $(\mathrm{N}=63)$ & Female $(\mathrm{N}=40)$ & $p$ value & Statistical significance \\
\hline Mean length of distal incus $(\mu \mathrm{m})$ & 1,200 & 1,200 & 0.16 & No \\
Mean area of distal incus $\left(\mu \mathrm{m}^{2}\right)$ & 590,000 & 520,000 & 0.04 & Yes \\
Mean diameter of long process $(\mu \mathrm{m})$ & 650 & 620 & 0.32 & No \\
Mean diameter of pedicle $(\mu \mathrm{m})$ & 270 & 230 & 0.07 & No \\
Mean diameter of plate $(\mu \mathrm{m})$ & 730 & 670 & 0.004 & Yes \\
\hline
\end{tabular}

Statistical significance is calculated using $t$ test and is defined as $p<0.05$ 
A recent histological investigation of the existence of a fourth ossicle in human ears by Palchun and Magomedov (1997) reaches a conclusion that is entirely contradictory to our results. Specifically, "Investigation of a series of histological sections of 40 auditory bones showed the existence of a dense partially developed connective tissue between the long process of the incus and the lenticular process. This indicates that the lenticular process is a separate bone - the least in the human body (weight $=0.12$ $0.20 \mathrm{mg}$, diameter $=0.5-0.8 \mathrm{~mm}$ )." (From English abstract of Palchun and Magomedov 1997)

How could observations of sections from 40 specimens not show a bony connection that we see signs of in 261 of 270 (97\%) well-preserved specimens and is completely defined in a single $20-\mu \mathrm{m}$ section from each of 108 of those 270 (40\%) specimens?

Differences in procedures may have contributed to the differences between these results and ours. We consider four possibilities:

1. Tissue dissection: "The incus was fixed in a $10 \%$ solution of neutral formalin with subsequent decalcification of the bone tissue in a $10 \%$ solution of hydrochloric acid." (Palchun and Magomedov 1997_-in Russian; this and subsequent quotes from this paper, unless specified, come from a translation by InTransCo. Inc. www.intransco.com). This statement suggests that each incus was excised from its ear before its histological preparation. This separation is consistent with the absence of the stapes in the paper's two histological images and with the paper's reference to the "free end" of the lenticular process. In contrast, in our preparations, the temporal bone was intact so that sections included the incus in-situ with its connections to the stapes and malleus.

2. Tissue embedding: "After total dehydration, specimens were embedded in paraffin ... serial sections (were produced) using a rotary microtome, with the thickness of sections being 5-7 $\mu \mathrm{m}$. The deparaffinized sections were stained with hematoxylin and eosin according to Van Giesen and impregnated with silver according to Gomori." In the Otopathology Laboratory at the Massachusetts Eye \& Ear Infirmary, celloidin sections are regarded as more robust and therefore preferable to paraffin (Schuknecht 1993). In our experience, even well decalcified bone will break and distort when cut in paraffin sections.

3. Alignment of section with the lenticular process: The plane of sectioning "passed through the long process of the incus its terminal section and the lenticular process." This description does not define an orientation for the plane of section. It seems likely that variations in orientation relative to the incus would produce sections that differ from one another in the particular structures encountered. Neither the process for selecting the key sections nor the number available for each incus is mentioned. Poor alignment of the plane of section with the lenticular process could decrease the likelihood of obtaining sections containing the bony pedicle connecting the distal long process and the plate. Such errors in alignment and the standard practice of only staining and inspecting every tenth $20-\mu \mathrm{m}$ section of the bones in our collection explained why we only see a complete bony connection between the distal incus and the lenticular plate in single sections from 108 of our 270 specimens.

4. Tissue identification: The Palchun and Magomedov study does not indicate the basis for identification of the connecting tissue as "cartilage"; the paper's English abstract, quoted above, calls it "dense, partially developed connective tissue". As this histological classification is the essential link in the conclusion, the precise criteria are crucially important.

Assessment of the possible importance of any of these factors on the results is speculative. Perhaps, through a combination of lost sections and misalignment of sections with the pedicle, the methods of the Palchun and Magomedov (1997) missed a bony connection of the size and shape seen in our material. In any case, the absence of a bony connection in the sections does not demonstrate its non-existence in the ear. On the other hand, the presence of a complete bony pedicle connecting the long process of the incus to the lenticular element in single sections from 108 of our 270 well-preserved specimens, together with signs of a bony pedicle in another 153 specimens in our study is strong support for a three-ossicle chain in human middle ears with a lenticular process (epiphysis) of the incus having two bony components (pedicle and plate) — across post-natal age.

2. The lenticular process consists of two distinct components: a proximal narrow pedicle and a distal flattened plate

The name "lenticular process" suggests that its shape is similar to that of a lentil. In many otologic texts, the lenticular process is pictured as a lentil-like disc, with the diameter of the distal long process being similar to the diameter of the lenticular process, as shown in Figure 1. In our histological material, the bony portion of the lenticular process consists of a narrow proximal pedicle and a flattened distal plate. The bony pedicle is surrounded on all sides by thick I-S joint capsular fibers, which is composed mainly of elastic fibers.

The presence of a narrow bony pedicle and a flattened plate of the human lenticular process has been described in the literature (see Funnell et al. 
2005, for history), but it has sometimes been considered an occasional or abnormal finding in human. In a study on the vascular supply of the incus, Alberti wrote "an incidental finding was that the neck of the lenticular process contains only a thin strut of bone, the bulk of this area is filled out by fibrous tissue" (Alberti 1965). In another study on normal and pathological incudes, Bellucci and Wolff (1966) showed a histological section of the distal incus with its narrow bony pedicle, and commented that the narrow pedicle was a result of "aseptic necrosis" (necrosis without any evidence of an infection). Similarly, the specialized I-S joint capsular fiber attachment to the bony pedicle has also been cited as pathologic: in a study on stapedectomy, Baron (1963) attributed the scalloping of the bony pedicle as evidence of avascular necrosis (he described it as "moth-eaten bone surfaces").

In the present study, we find that the narrow bony pedicle of the lenticular process is a regularly occurring feature of the normal anatomy. The narrow bony pedicle and expanded plate configuration of the lenticular process is present in 107/108 normal temporal bone preparations across a wide age range. This configuration of the lenticular process is present at birth, and remains largely unchanged with age. The slight scalloping of the bony pedicle is likely a result of I-S joint capsular fibers attaching directly onto the bony surface and is not due to bone resorption.

\section{Implications of the anatomy of the distal incus and lenticular process on middle-ear mechanics}

What effect might the pedicle and plate structures of the lenticular process and the configuration of the I-S joint capsule have on middle-ear mechanics? More specifically, how might the narrow bony pedicle with the surrounding capsule influence coupling of ossicular motion from incus to stapes? One possibility is that there is bending of the bony pedicle, and that such flexibility of the pedicle helps to isolate the stapes from some components of incus motion, as illustrated in Figure 9. The narrow pedicle may bend easier with vertical forces that push superiorly and inferiorly or horizontal forces that push anteriorly and posteriorly, compared to forces that push laterally and medially. Such bending of the pedicle would allow the stapes to move in a piston-like lateral-to-medial direction when stimulated by the rotating incus, while any superior-to-inferior motions, or anterior-to-posterior motions, are reduced by bending of the pedicle before they are transmitted to the stapes head (Funnell et al. 2005; Decraemer and Khanna 2004). The elastic fiber component of the I-S joint capsule itself might also be expected to contribute to this process.

Funnell et al. (2005) proposed a finite element model of the elastic behavior of the lenticular process in

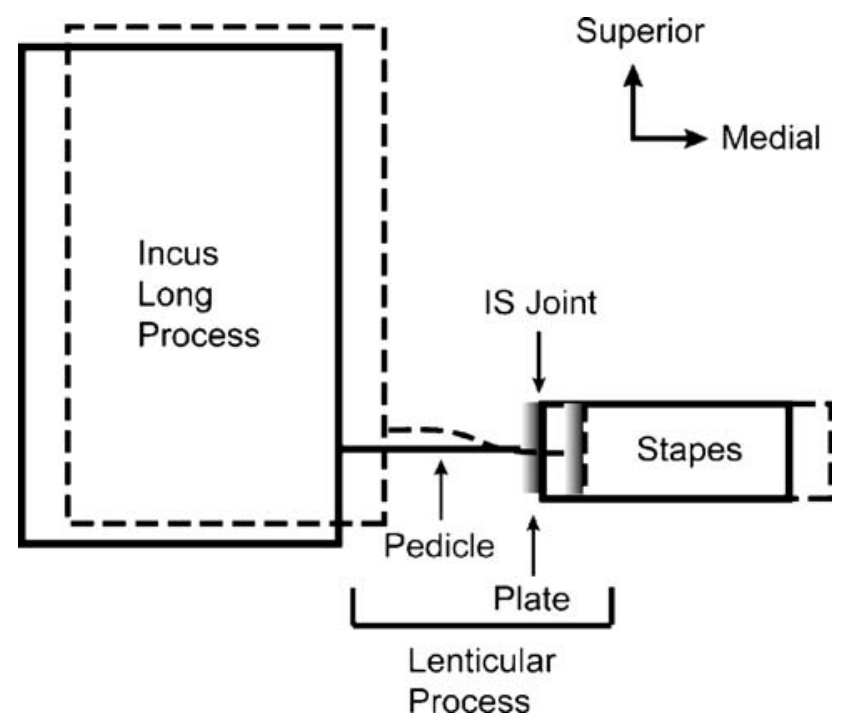

FIG. 9. Schematic of the I-S joint at rest (solid line) and displaced (dashed line). The flexibility of the pedicle helps to isolate the stapes from the upward, downward, and sideward motion of the incus while faithfully translating piston-like lateral-to-medial motions from incus to stapes.

cat ears, based on their anatomical observations. Their analysis demonstrates that the thin pedicle in their model can bend significantly thereby adding flexibility to the I-S joint and reducing the transmission of particular motion components from the incus to the stapes.

One approach to testing assumptions that couple anatomy to physiology is to test the hypotheses for simple physiological conditions. Funnell and co-workers' purely elastic model of the ossicles and pedicle can be tested by studies of the three-dimensional sound-induced motion of the incus and stapes with special attention to differences in motion between the long process of the incus and the head of the stapes. Measurements of the relative motion of the incus and stapes head produced by stapedial muscle contraction (Pang and Peake 1986) or low-frequency sound (Guinan and Peake 1967) have demonstrated little relative difference in the medial-tolateral piston-like motions of the incus and stapes produced by sound stimulation, and prominent sliding between the incus and stapes head with the anterior-toposterior motions produced by stapedius muscle contraction. These and more recent three-dimensional motion studies (e.g., Decraemer and Khanna 2004) can be used to test structural models that include a thin and possibly flexible lenticular pedicle.

\section{Features of the anatomy of the distal incus and lenticular process may help to explain the vulnerability of this region in middle-ear pathology}

Our study suggests two factors that could contribute to the pathologic resorption of bone in this region: (1) the slenderness of the bony pedicle of the lenticular process and (2) the progressive loss of 
osteocytes observed with aging. The bony pedicle of the lenticular process is only $0.26 \mathrm{~mm}$ on average in diameter, making it one of the thinnest parts of the ossicular chain. Thus, resorption of even a small amount of bone at the level of the pedicle would be sufficient to interrupt the bony connection between the incus long process and the lenticular plate and thereby alter sound transmission through the ossicular chain. Our clinical observations (SNM) during middle-ear surgery for chronic otitis media indicate that, in cases with incus resorption, the pedicle is often missing, while the plate of the lenticular process is usually preserved, which is consistent with the notion that the pedicle is vulnerable to bone resorption because its bone is thin. Progressive loss of osteocytes with age (Fig. 8) has been reported for all three ossicles (Marotti et al. 1998). Osteocytes play a crucial role in bone remodeling (Burger and KleinNulend 1999). It is possible that loss of osteocytes in the ossicles increases the susceptibility of thinner areas of the ossicular chain (such as the ossicular pedicle) to bone resorption.

\section{Incus necrosis and blood supply}

"Necrosis" of the incus is a complication of stapedectomy (stapedectomy is a procedure where a prosthesis is coupled to the ossicular chain by wrapping a metal wire or band around the distal incus). It has been suggested (Smyth 1964) that tightening the prosthesis wire excessively may interfere with the incus' 'tenuous' blood supply and cause bony necrosis. We found that the distal incus, including the lenticular process, has in fact, a vascular network that included several blood vessels coursing within the bone, a mucosal plexus on the outer surface of the bone, and frequent communications between the two vascular systems. Our description of the blood supply of this region is similar to that reported by Nager and Nager (1953) and Alberti (1965). Given the abundant vascular network in this region, it seems unlikely that incus necrosis is caused by a lack of blood supply.

\section{CONCLUSION}

The anatomy of the distal incus is more complex than standard descriptions in otology texts. The bony portion of the lenticular process has a proximal narrow pedicle and a distal flattened plate. The capsule of the I-S joint is specialized; it attaches to a narrow area along the rim of the stapes head, expands considerably at the level of the pedicle, and is attached to the pedicle and plate over a broad surface area. It has a considerable component of elastic tissue. These structures are apparently full size at birth. These anatomical features have implications for basic mechanisms of middle-ear sound transmission and for the vulnerability of the distal incus to resorption in middle-ear disease.

\section{ACKNOWLEDGEMENTS}

We thank Diane Jones, Barbara Burgess, Jennifer O’Malley, Richard Cortese, and Michael E. Ravicz for their professional contributions. Funded in part by NIDCD (grants R01 DC04798, U24 DC008559, and T32 DC000020), Mr. Axel N. Eliasen, Mr. Lakshmi Mittal, and the Silverstein Young Investigator Award (to WC).

\section{REFERENCES}

AlBERTI PW. The blood supply of the long process of the incus and the head and neck of stapes. J. Laryngol. Otol. 79(11):966-970, 1965.

BARON SH. Persistent stapedial artery, necrosis of the incus, and other problems which have influenced the choice of technique in stapes replacement surgery in otosclerosis. Laryngoscope 73:769-782, 1963.

Bellucci RJ, WolfF D. The incus, normal and pathological. Arch. Otolaryngol. 83(5):413-419, 1966.

BuRger EH, Kuein-Nulend J. Mechanotransduction in bone-role of the lacunocanalicular network. FASEB J. 13(Suppl):S101-S112, 1999.

DAviES DV. A note on the articulations of the auditory ossicles. J. Laryngol. Otl. 62:533-538, 1948

Decraemer WF, Khanna SM. Measurement, visualization and quantitative analysis of complete three-dimensional kinematical data sets of human and car middle ear. In: Gyo K, Wada H, Hato N, Kooike T (eds) Middle Ear Mechanics in Research and Otology. Singapore, World Scientific, pp. 3-10, 2004.

Funnell WR, Heng Siah T, McKee MD, Daniel SJ, Decraemer WF. On the coupling between the incus and the stapes in the cat. J. Assoc. Res. Otolaryngol. 6(1):9-18, 2005.

Funnell WRJ, Daniel SJ, Alsabah B, Liu H. On the coupling between the incus and the stapes. Auditory mechanisms: Processes and models. Proc. Ninth International Symposium, Portland (OR), 115-116, 2006.

Glasscock ME, Gulya AJ. Glasscock-Shambaugh Surgery of the Ear. 5th edn. Ontario, B. C. Decker, 2005.

Guinan JJ, JR, Peake WT. Middle-ear characteristics of anesthetized cats. J. Acoust. Soc. Am. 41(5):1237-1261, 1967.

HaRTy M. Elastic tissue in the middle-ear cavity. J. Laryngol. Otol. 67:723-729, 1953

LunA LG. Histopathologic Methods and Color Atlas of Special Stains and Tissue Artifact. Gaithersburg, American Histolabs, 1972.

Marotti G, Farneti D, Remaggi F, Tartari F. Morphometric investigation on osteocytes in human auditory ossicles. Ann. Anat. 180(5):449-453, 1998.

NAGER GT, NAGER M. The arteries of the human middle ear, with particular regard to the blood supply of the auditory ossicles. Ann. Otol. Rhinol. Laryngol. 62:923-949, 1953.

Palchun VT, Magomedov MM. Some anatomical features of the long process of the incus. Vestn. Otorinolaringol. 2:19-20, 1997, (in Russian: Translated by InTransCo. Inc., Lynnfield, MA, USA, www.intransco.com).

Pang XD, Peake WT. How do contractions of the stapedius muscle alter the acoustic properties of the ear? In: Allen JB, Hall JL, Hubbard A, Neely ST, Tubis A (eds) Peripheral Auditory Mechanisms. New York, Springer, pp. 36-43, 1986. 
Ross MH, Pawlina W. Histology: A Text and Atlas. 5th edn. Philadelphia, Lippincott Williams \& Wilkins, 2005.

Schuknecht HF. Pathology of the Ear. 2nd edn. Philadelphia, Lea \& Febiger, 1993.

SharpNell HJ. On the Structure of the Os Incudi. London Med Gazette, pp. 171-173, 1832
SMYTH GD. The long process of the incus. J. Laryngol. Otol. 78:400407,1964

Valsalva AM. De Aure Humana Tractatus, Bononiae, Bologna, 1704.

Wolff D, Bellucci RJ, Eggston AA. Surgical and Microscopic Anatomy of the Temporal Bone. New York, Hafner, 1971. 